\title{
Impact on Early Discharge After Emergency Department-Based Treatment Pathway in Nonperforated Appendicitis: A Retrospective Study
}

\author{
Artur Ojakäär ( $\nabla$ artur.ojakaar@hus.fi ) \\ Kanta-Hameen keskussairaala https://orcid.org/0000-0002-9569-2753 \\ Martin Purdy \\ Kanta-Hameen keskussairaala
}

Aristotelis Kechagias

Kanta-Hameen keskussairaala

Ulla Järvelin

Tampereen yliopistollinen sairaala

Ari Palomäki

Kanta-Hameen keskussairaala

Research article

Keywords: appendicectomy, appendectomy, day surgery, reorganisation, emergency department

Posted Date: February 14th, 2020

DOI: https://doi.org/10.21203/rs.2.23565/v1

License: (c) (i) This work is licensed under a Creative Commons Attribution 4.0 International License. Read Full License 


\section{Abstract}

\section{Background}

Acute appendicitis is a global disease and a very common indication for emergency surgery worldwide. The need for hospital resources is therefore constantly high. The administration in Kanta-Häme Central Hospital, southern Finland, called for an urgent reorganisation due to shortage of hospital beds at the department of general surgery. Treatment pathway of nonperforated acute appendicitis was moved into Emergency Department (ED). The aim of this study was to assess whether it affected the length of inhospital stay (LOS) and the 30-day complication rate.

\section{Methods}

This is a retrospective pre- and post- intervention analysis. After the reorganisation, most postoperative patients with nonperforated appendicitis were followed at the 24-hour observation unit of the ED instead of the surgical ward. Patients operated during the first three months after the reorganisation were compared to those operated during the three months before it. A case met inclusion criteria if there were no signs of appendiceal perforation during surgery. Exclusion criteria comprised age $<18$ years and perforated disease.

\section{Results}

During the study period, appendicectomy was performed on 112 patients, of whom 62 were adults with nonperforated appendicitis. Twenty-seven of the included patients were treated before the reorganisation, and 35 after it. Twenty of the latter were followed only at the ED. LOS decreased significantly after the reorganisation. Median postoperative time till discharge was $15.67 \mathrm{hrs}$ for all patients after the reorganisation and $13.13 \mathrm{hrs}$ for those exclusively followed at the ED, compared to $24.42 \mathrm{hrs}$ before the reorganisation (standard error $6.2 \mathrm{hrs}, 95 \%$ confidence interval $2.3-15.2 \mathrm{hrs}, \mathrm{p}<0.01$ ). There were no more complications in the group treated postoperatively in the ED.

\section{Conclusions}

Early discharge from the ED observation unit after appendicectomy for nonperforated appendicitis in adults is safe and feasible. Shifting the postoperative monitoring and the discharge policy of such patients to the ED - instead of the surgical ward - occurred in the majority of the cases after the reorganisation. This change may spare resources as in this series it resulted in a significantly shorter LOS without any increase in the 30-day complication rate. 


\section{Introduction}

Discharge time after operation of uncomplicated appendicitis has been significantly shortened in the last 35 years. In the 1980s there was an average discharge at 3-4 days postoperatively (1). Since the 1990s, it has been a standard practice to discharge patients within $24-48$ hours $(2,3)$. The development of laparoscopic surgery further improved early discharge rates after appendicectomy without additional complications (4).

Kanta-Häme Central Hospital $(\mathrm{K}-\mathrm{HCH})$ is a secondary care hospital located at South of Finland. The Emergency Department (ED) of K-HCH has modern facilities and a subunit dedicated to the short-term ( $\leq$ $24 \mathrm{hrs}$ ) observation of patients with an acute condition (5). In addition, emergency medicine has been introduced as a separate specialty branch in Finland in 2013 (6). Since then, there has been a continuous reappraisal and development of its functionality within the inter-disciplinary management of patients, such as those operated for an acute abdominal condition (7-10).

A recent administrative decision of $\mathrm{K}-\mathrm{HCH}$ called for an active participation of the $\mathrm{ED}$ in the in-hospital management of adult patients with acute appendicitis. The intent was to reduce the use of hospital space at the department of general surgery within a few weeks. This included a reorganisation according to which the cases with nonperforated appendicitis would be followed preferentially at the ED during the pre- and post- operative period. For this reason, a new protocol for a same-day patient discharge was implemented in the facilities of the ED.

The objective of this retrospective study is to analyse the immediate effect of this new protocol on the length of stay (LOS) of adult patients operated for nonperforated acute appendicitis and on the 30-day complication rate. Our special interest is to assess whether LOS can be safely shortened when patients are treated pre- and post- operatively at the ED instead of the surgical ward.

\section{Material And Methods}

We conducted a retrospective study on all adult patients undergoing appendicectomy for suspected acute appendicitis during a six-month period. Data were gathered from the electronic patient record system and from the operation registry of the K-HCH. Patients with the Nordic Medico-Statistical Committee (NOMESCO) code JEA00 or JEA01 for open and for laparoscopic appendicectomy were included. Clinical suspicion or radiological diagnosis of acute appendicitis were the indications for surgery.

We included patients at least 18 years old. Patients with normal appendix were excluded from the analysis. An intra-operative scoring system was used to grade the severity of appendicitis (Table 1) (11). Patients with grade la to Ic appendicitis without perforation or any other significant complications were included in the analysis. 
We retrospectively compared the postoperative stay and LOS of patients with acute nonperforated appendicitis. The results over the period of three months after the reorganisation were compared to those over the three months before the reorganisation. Before the reorganisation, patients were always followed at the department of surgery, which included at least one clinical visit from a surgical consultant or resident on the first postoperative morning. According to the reorganisation, a patient with surgery for nonperforated disease would be preferentially followed at the ED with an intent of same-day discharge in the case of an uneventful postoperative course. The ED nurses had the duty for the continuous assessment of the patient's clinical status postoperatively, and the doctor on-call (in most cases an earlycareer ED resident) had the responsibility of the discharge. Therefore, the reorganisation shifted the discharge process to the ED after surgery for nonperforated appendicitis, with surgical consultation only on demand.

We documented complications and readmissions for 30 days postoperatively. Patients receiving any operation at our institution are referred to us for any complication that cannot be managed at the level of the general practitioner, according to a strict referral system. The demographics of the patients treated before and after the reorganisation of the process were compared. Moreover, we analysed time of presentation (during office hours vs. out-of-office hours), physical status based on the classification of the American Society of Anesthesiologists (ASA) and surgical technique (laparoscopic vs. open surgery).

\section{Statistical Methods}

We performed statistical analyses using IBM ${ }^{\circledR}$ SPSS ${ }^{\circledR}$ Statistics Version 24 (copyright 2016). Data are presented as medians (minimum-maximum, interquartile range) in cases of non-normality. We assessed differences in continuous variables using the Mann-Whitney U-test. Differences in dichotomous variables were analysed using the Chi- square test or Fischer's exact test as appropriate. A probability value $<0.05$ was considered significant.

\section{Results}

During the study period, a total of 112 patients with acute appendicitis were operated on. Sixty-two $(61.4 \%)$ adult patients with nonperforated appendicitis were included in the analysis (Fig. 1).

Of the patients evaluated, 27 were women and 35 men. The difference in gender between the groups treated before and after the reorganisation of our process was not significant. Median age of patients was 33 years (min - max: 18-78) with no significant differences between the groups (NS). Patients' characteristics are presented in Table 2. Further, there were neither any significant differences concerning $\mathrm{BMI}, \mathrm{ASA}$ class or use of laparoscopic operation technique. Patients were also admitted to the hospital during office-hours similarly before and after the reorganisation (Table 2).

Before the reorganisation, all of 27 patients (100\%, Group 1) were followed postoperatively at the surgical ward. Immediately after the start of the reorganisation (Group 2), 20 patients (57.1\%) were followed at the 
ED observation unit and 15 (42.9\%) at the surgical ward. The results concerning postoperative stay in hospital are presented in Fig. 2. The median postoperative length of stay decreased significantly by $46.2 \%$, being $24.42 \mathrm{hrs}$ and $15.67 \mathrm{hrs}$ before and after the reorganisation respectively. When only patients in Group 2 (after the reorganisation) were compared, the median postoperative LOS among patients treated postoperatively at the surgical ward and at the ED-only were $25.03 \mathrm{hrs}$ and $13.13 \mathrm{hrs}$ respectively.

The ASA classification of 20 patients discharged from the ED was as follows: 14 patients in ASA 1, five in ASA 2 and one in ASA 3. One patient in Group 2 who was treated postoperatively on the surgical ward had a superficial wound infection at 30-day follow-up (NS). All other patients in Groups 1 and 2 remained free from postoperative complications. There were no readmissions.

\section{Discussion}

In this study, we evaluated the length of postoperative hospital stay in patients operated on for nonperforated acute appendicitis after a reorganisation oriented to same-day discharge. We showed that LOS was significantly shorter in patients treated at the ED than among those treated at the surgical ward postoperatively. The postoperative direction of the patient to the ED was successful in the majority of the cases after the reorganisation, despite the fact that the time to implement the new protocol for patient management was only a few weeks. This corresponds to a rather satisfactory initial compliance of the medical and nursery staff in the implementation of the new protocol.

Our findings concur with earlier studies, where early discharge of patients with non-perforated acute appendicitis was reported to be safe and effective $(12,13)$. A review of 13 studies with 1152 adult patients who underwent day-case appendicectomy reported that only $27 \%$ were discharged within 12 hours, $53 \%$ within 24 hours, and $21 \%$ within 72 hours (14). The cases of our study that were followed at the ED after the reorganisation (57.1\%) approached a discharge within 13 hours from the operation. Thus, the majority of our Group 2 patients virtually reached the most favourable benchmark of the aforementioned review (14). Interestingly, there are further studies from the United States and Canada that have reported impressive results with a postoperative discharge within 3-4.7 hours in the majority of their series $(45-86 \%)(12,13)$. This was associated with a return-rate to the ED of $8-11.4 \%$ (the respective rate was $1.6 \%$ in our series) without any need for in-hospital re-admission $(12,13)$. The Canadian study showed that the early-discharge policy resulted in a $45 \%$ reduction in the need for inhospital beds (13). Same-day discharge is therefore feasible and effective. Possibly the variation in the length of postoperative stay can be attributed to modifiable local factors and results can be further improved with extensive training (15). In addition, early discharge is feasible and reliable also in paediatric patients (16).

An important aspect of the herein research is that it provides real-life results concerning early discharge after surgery for nonperforated acute appendicitis. To the best of our knowledge there is scarce evidence concerning early- or same day- discharge from the Scandinavian Countries and particularly from Finland. It is interesting to note that the main proponent of non-operative management for nonperforated acute 
appendicitis, which is the APPAC multi-centre randomised trial from Finland between 2009-2012, did not take into account the potential benefits of early discharge to the comprehensive outcome. According to the APPAC conservative therapy with antibiotics was non-inferior to surgery (17). At 5 years the majority $(61 \%)$ of antibiotic group patients did not undergo appendicectomy and the overall costs of the surgical arm were 1.4 times higher $(18,19)$. No information was provided concerning the time from surgery to discharge (17). Moreover, in the surgery group, only standard open appendicectomy was performed and possibly this may have contributed to a delayed discharge compared to modern laparoscopic appendicectomy, even in complicated cases (20). On the contrary, it has been shown that early discharge after appendicectomy confers a significant reduction in the costs (21). We believe that the results of our study should be taken into account in this context, as the overall benefit of non-operative treatment for nonperforated acute appendicitis could be challenged from an aggressive early discharge policy after laparoscopic appendicectomy.

The potential weaknesses of a study with a retrospective design are contained in this series as the major end-points are electronically recorded during the routine clinical practice at our institution. The time of surgery and the time of discharge are always digitally documented on the spot. In addition, there is a valid electronic documentation of the 30-day complications due to a strict post-discharge policy that comprises direct telephone consultation from the patient's family doctor and referral for complication management to our institution. On the other hand, there is a possibility of a selection bias given the fact that there was not a robust compliance to the reorganisation's policy, at least during the initial 3-month period. Indeed, 15 out of the 35 patients were directed to the surgical ward instead of the ED, possibly due to the surgeons' preference not to deviate from the previous routine (15). However, this study shows that a major change in routine practice is feasible even at a short time and at a satisfactory compliance rate, and with immediate positive outcome in respect of the initial objective. Another interesting feature of the herein investigation, is that the responsibility of the postoperative course of patients operated for nonperforated acute appendicitis can be safely assigned to a non-surgical specialty such as emergency medicine, and even at the level of an early-career house officer. It is also possible that further reorganisation of the whole in-hospital management, and particularly a more active role of the recovery room to the early discharge, could result in an even shorter LOS than the one documented in this study $(21,22)$. Last, this study confirmed that surgery for nonperforated appendicitis bears minimal postoperative morbidity as there was not any significant complication in all the series. Therefore, despite the moderate sample-size we believe that this series is not statistically underpowered to support our secondary end-point findings that early discharge does not affect post-operative complication and readmission rate.

\section{Conclusions}

In this retrospective study of adult patients with acute nonperforated appendicitis, LOS was significantly shorter in patients who were followed postoperatively at the ED than at the surgical ward. This reflects the feasibility and the positive impact of the implementation of a same-day discharge protocol. Moreover, the 
shifting of the discharge process and responsibility to a non-surgical unit such as the ED proved to be functional and safe.

\section{List Of Abbreviations}

ASA American Society of Anesthesiologists

BMI Body mass index

ED Emergency Department

K-HCH Kanta-Häme Central Hospital

LOS Length of stay

NOMESCO Nordic Medico-Statistical Committee

\section{Declarations}

\section{Ethics approval and consent to participate}

This was a retrospective pre- and post-intervention analysis, none of the patients were contacted. The Regional Ethics Committee of Tampere University Hospital (TAYS) approval was applied.

\section{Consent for publication}

Not applicable.

\section{Availability of data and materials}

Not applicable.

\section{Competing interests}

The authors declare that they have no competing interests.

\section{Funding}

This study was funded by The Ministry of Health and Social Welfare in Finland via the Medical Research Fund of Kanta-Häme Central Hospital and Pirkanmaa's Hospital District.

\section{Authors' contributions}

MP and AP designed the study; MP and AO organised the data collection; AP carried out the statistical analyses; all authors for their part drafted and critically revised the manuscript, and, finally, read and approved it. 
Acknowledgements

The authors gratefully acknowledge the professional linguistic aid of Virginia Mattila. We also wish to express our thanks to the medical and nursing staff working in the ED as well as in the operation unit and the unit of gastroenterological surgery.

\section{References}

1. Vinz H, Moltrecht S, Müller K, Reisig J, Grobler B, Golombek F. Early postoperative discharge. Zentralbl Chir. 1981;106:985-91.

2. Lord RV, Sloane DR. Early discharge after open appendicectomy. Aust N Z J Surg. 1996;66:361-5.

3. Ramesh S, Galland RB. Early discharge from hospital after open appendicectomy. Br J Surg. 1993;80:1192-3.

4. Macarulla E, Vallet J, Abad JM, Hussein H, Fernández E, Nieto B. Laparoscopic versus open appendectomy: a prospective randomized trial. Surg Laparosc Endosc. 1997;7:335-9.

5. Lehtonen $H$, Lukkarinen $T$, Kämäräinen V, Rautava V-P, Parviainen P, Palomäki A. Improving Emergency Department Capacity Efficiency. Signa Vitae. 2016;12:52-7.

6. Naskali J, Palomäki A, Harjola V-P, Hällberg V, Rautava V-P, Innamaa T. Emergency Medicine in Finland: First Year Experiences of Specialist Training. Acad J Emerg Med. 2014;13:26-9.

7. Heikkilä I, Kuusisto H, Stolberg A, Palomäki A. Stroke thrombolysis given by emergency physicians cuts in-hospital delays significantly immediately after implementing a new treatment protocol. Scand J Trauma Resusc Emerg Med. 2016;24:46.

8. Rautava V-P, Palomäki E, Innamaa T, Perttu M, Lehto M, Palomäki A. Improvement in self-reported confidence in nurses' professional skills in the emergency department. Scand J Trauma Resusc Emerg Med 2013;21:16.

9. Saarinen HJ, Palomäki A. Acute renal infarction resulting from fibromuscular dysplasia: a case report. J Med Case Rep. 2016;10:118.

10. Heikkilä I, Kuusisto H, Holmberg M, Palomäki A. Fast Protocol for Treating Acute Ischemic Stroke by Emergency Physicians. Ann Emerg Med 2019;73:105-12.

11. Guzmán-Valdivia Gómez G. Una clasificación útil en appendicitis aguda [A useful classification for acute appendicitis]. Rev Gastroenterol Mex. 2003;68:261-5.

12. Hrad V, Waisbren SJ. Results of immediate discharge from postanesthesia care unit to home after laparoscopic appendectomy for acute nonperforated appendicitis. Surg Laparosc Endosc Percutan Tech.2015;25:343-6

13. Dubois L, Vogt KN, Davies W, Schlachta CM. Impact of an outpatient appendectomy protocol on clinical outcomes and cost: a case-control study. J Am Coll Surg. 2010;211:731-7.

14. Cosse C, Sabbagh C, Grelpois G, Brehant O, Regimbeau J M. Day case appendectomy in adults: a review. Int J Surg. 2014;12:640-4 
15. Aguayo P, Alemayehu H, Desai AA, Fraser JD, St Peter SD. Initial experience with same day discharge after laparoscopic appendectomy for nonperforated appendicitis. J Surg Res. 2014;190:93-7.

16. Benedict LA, Sujka J, Sobrino J, Aguayo P, St Peter SD, Oyetunji TA. Same-day discharge for nonperforated appendicitis in children: an updated institutional protocol. J Surg Res. 2018;232:346350

17. Salminen P, Paajanen $H$, Rautio T, et al. Antibiotic therapy vs appendectomy for treatment of uncomplicated acute appendicitis: the APPAC randomized clinical trial. JAMA. 2015;313:2340-8

18. Salminen P, Tuominen R, Paajanen $\mathrm{H}$, et al. Five-year follow-up of antibiotic therapy for uncomplicated acute appendicitis in the APPAC randomized clinical trial. JAMA. 2018;320:1259-65

19. Haijanen J, Sippola S, Tuominen R, et al. Cost analysis of antibiotic therapy versus appendectomy for treatment of uncomplicated acute appendicitis: 5-year results of the APPAC randomized clinical trial. PLoS One. 2019;14:e0220202

20. Quah GS, Eslick GD, Cox MR. Laparoscopic appendicectomy is superior to open surgery for complicated appendicitis. Surg Endosc. 2019;33:2072-82

21. Farach SM, Danielson PD, Walford NE, Harmel RP Jr, Chandler NM. Same-day discharge after appendectomy results in cost savings and improved efficiency. Am Surg. 2014;80:787-91

Mitchell MB, Daniel D. Homeward bound: the safety of discharging postappendectomy patients directly home from the recovery room. Am Surg. 2014;80:1069-73.

\section{Tables}

Table 1. Intra-operative scoring of appendicitis (11)

\section{Grade}

$0 \quad$ No appendicitis

Ia Oedematous, ingurgitated appendix

Ib Abscessed or phlegmonous appendix, presents fibrin membranes and seropurulent liquid around appendix

Ic necrosed appendix with no perforation

II perforated appendix with localized abscess

III complicated appendicitis with generalized peritonitis 
Table 2. Patients' demographics and characteristics before and after the reorganisation of the treatment process.

Variable

Number of patients

Age, median

Gender (men / women)

Body mass index, mean (SD)

Presentation during office hours* 13

ASA classification 1-2/3

Laparoscopic surgery

*Office hours: Monday to Friday, 8AM-4PM
24 / 331 / 4 N.S.

$1925 \quad$ N.S.

Figures 


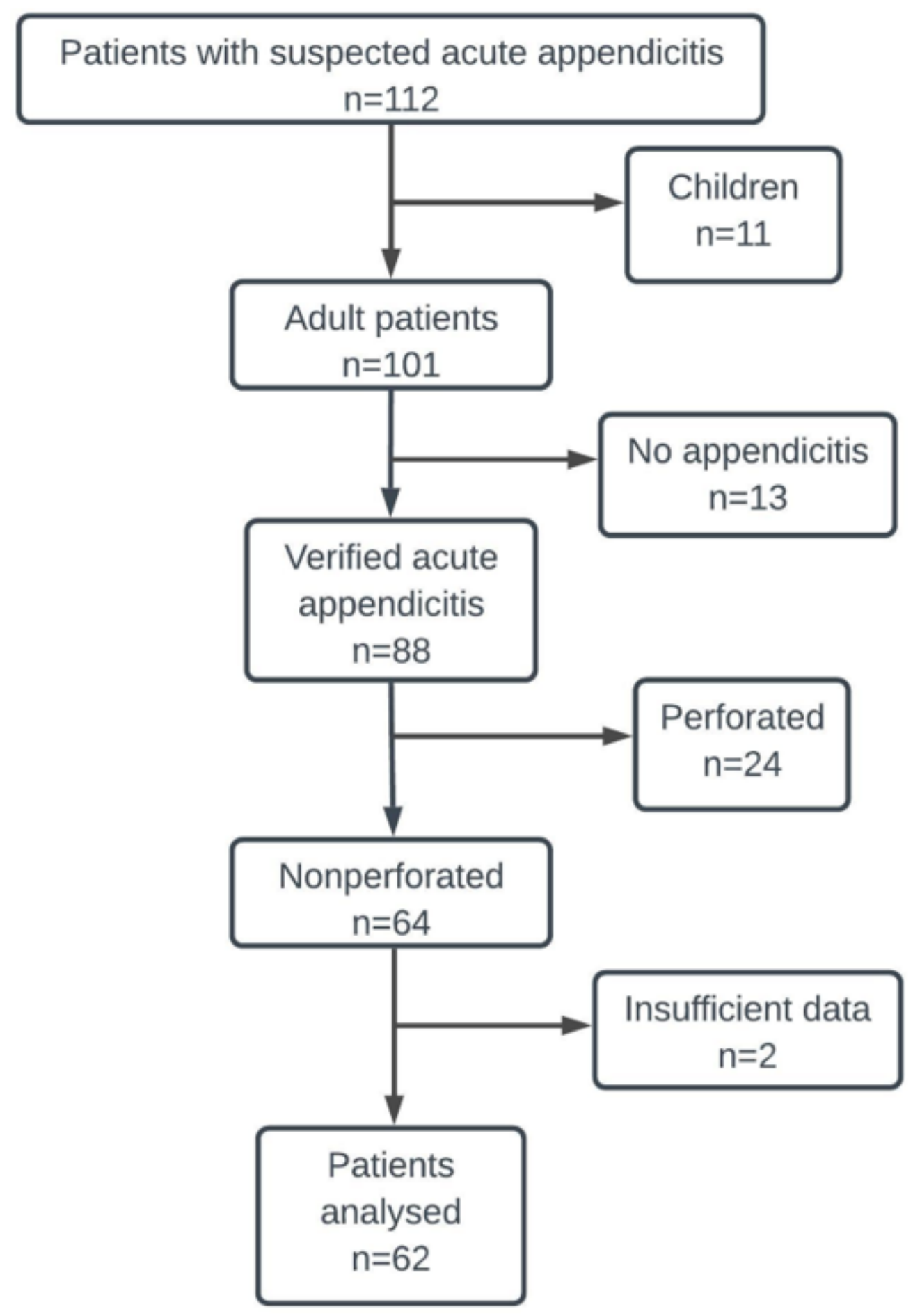

Figure 1

Flowchart of the study. 


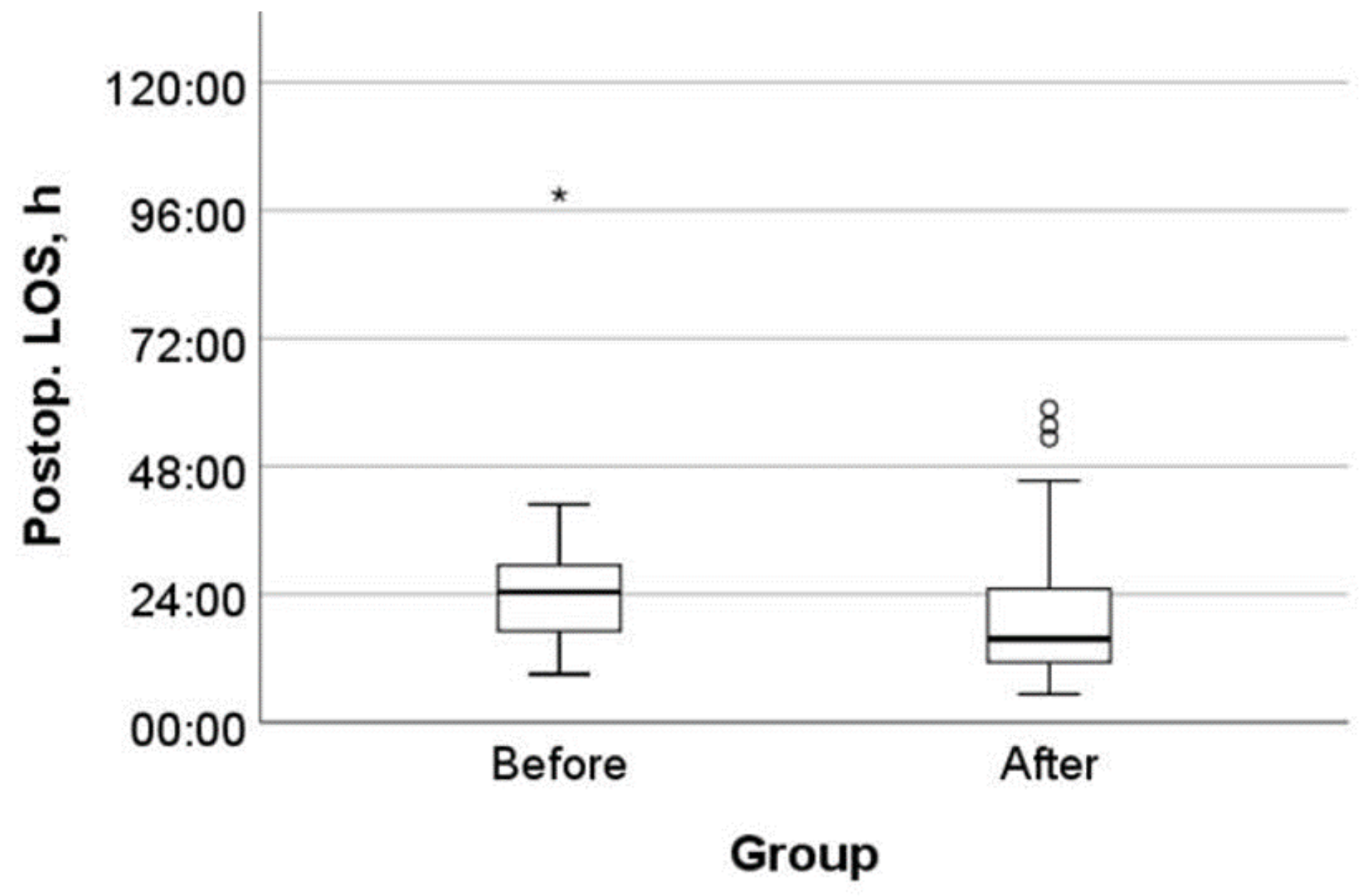

Figure 2

Postoperative length-of-stay before and after the reorganisation. 